\title{
An Empirical Investigation of Learning Curve Laws in the Tile Manufacturing Industry
}

\author{
A. P. Chatzimichali, V. D. Tourassis \\ Department of Production Engineering and Management, \\ Democritus University of Thrace, Xanthi, Greece
}

\begin{abstract}
The ceramic tile manufacturing industry is under constant strain today due to the competitive environment worldwide and to the changing patterns of consumer behavior. Despite its highly automated production lines, the industry is becoming less of a mass manufacturer and more of a batch producer with all the corresponding issues that such a move entails. The frequent setup and retooling of the production has led to renewed interest on the subject of learning curve laws and their impact on the productivity of items re-introduced after short periods of time to the product mix. The actual industrial data presented in this paper support the premise that the learning curve phenomenon is present, albeit in a more complex sense, when tile patterns are produced in relatively small batches and the resulting ramp-up is reminiscent of new product setup.
\end{abstract}

Keywords - Learning curves, tile manufacturing, production line setup

\section{INTRODUCTION}

Keeping up with the rapid pace of change in today's market can be a daunting task for firms. Companies try to launch new products faster in order to be competitive and take a bigger market share. To respond to this challenge greater variety of products and new designs are continuously developed, time-to-market is reduced and product lifecycle shrinks. In this environment of frequent product and process changes, the interest in the field of production learning is growing. Indeed, many researchers feel that the only sustainable advantage an organization will have in the future is its ability to learn faster than its competitors. [1].

The learning phenomenon has been widely studied in many fields and different models have been proposed based on theoretical or empirical approaches [2]. The practical consequence of organizational learning is that the time required to produce a unit decreases with the total number of units produced [3]. The learning curve depicts graphically the fact that production time decreases with cumulative production at a uniform rate [4]. Production ramp-up, on the other hand, is the period of time during which a manufacturing process is scaled up from a small, laboratory-like environment to a highvolume production setup [5]. Thus production ramp-up represents the time stage between product development and actual manufacturing [2].
In this paper, actual industrial data from a specific tile production facility are processed in order to assess the behavior of small-batch production in relatively welltuned production lines. The long-term objective is to model the line behavior and to develop an optimum batch production level for each tile pattern.

The proposed approach is initially vetted through the Franceshini-Galetto model [2,6] that proposes a new approach for predicting complex system behavior over time. This paper studies the learning and ramp-up curves using empirical data in order to predict the line behavior. Ceramic tiles exhibit non-normative behavior in their production process and thus complicate the development of adequate models. Indeed, even slight variations of the manufacturing process or the decoration of the product can cause serious chromatic defects and significant waste (production loss).

\section{PRODUCTION PROCESS DESCRIPTION}

Ceramic tile manufacturing nowadays is highly automated. Raw material consists of mined clay minerals which are wet milled and mixed. The excess water is removed using spray drying and the resulting powder is pressed into the desired tile body shape (dry pressing method). After forming, ceramic tiles are dried in continuous driers using hot air and moved to the glaze line. Manufacturers typically use two basic methods to glaze tiles as they move on the line, depending upon the tile pattern. In centrifugal glazing (decoré), the glaze is fed inside a barrel that rolls and flings the glaze onto the tile's surface. The bell/waterfall method is an alternative method where a stream of glaze falls onto the tile as it passes on a conveyor.

The next step in the process is heat treatment. Continuous kiln (tunnel kiln) is typically used; the tiles enter at one end, are gradually heated as they progress slowly towards the center of the kiln, and then are cooled slowly as they approach the opposite end of the tunnel. Finally, the production crew sorts the tiles into quality batches and scrap. The tiles are then automatically packed, palletized and shipped to the market. 


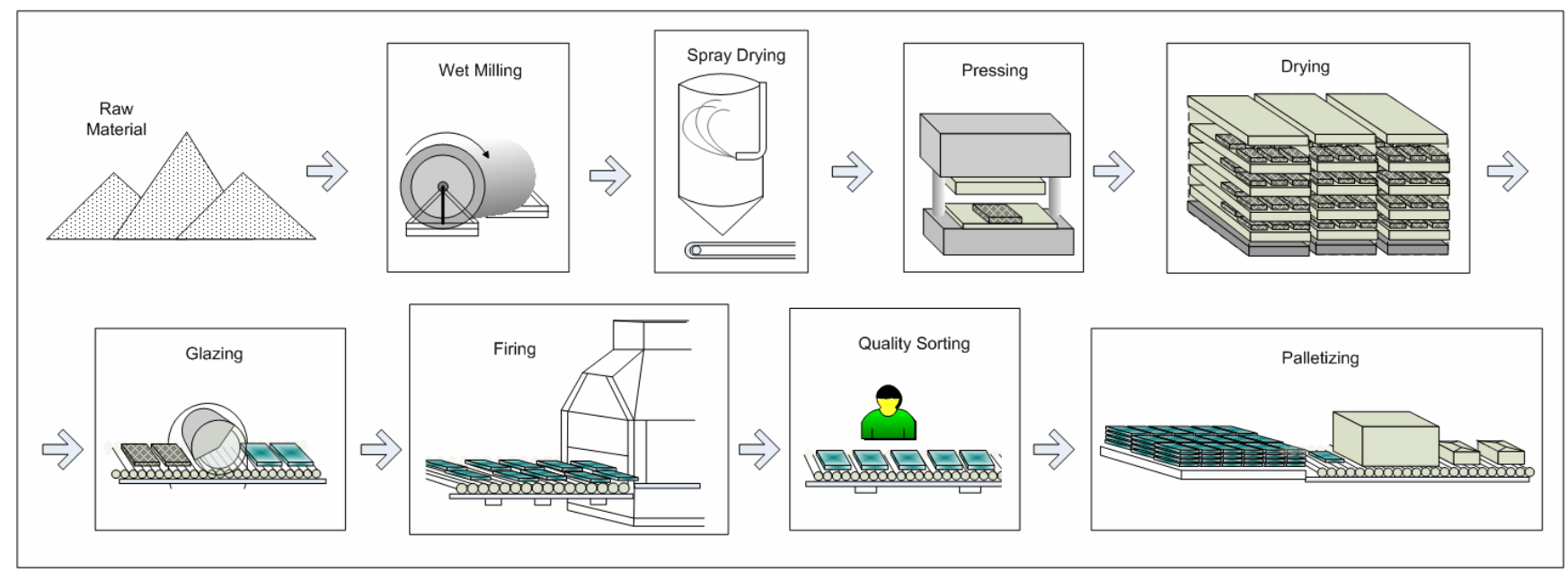

Fig. 1 - Production Process

The tile production process is fully automated and the tiles exhibit continuous flow on a conveyor belt. Due to the fact that drying kilns require a round-the-clock, constant flow of tiles in order to achieve maximum thermal efficiency, sufficient buffers are often employed just before the kilns. Fig. 1 provides a graphical representation of the tile manufacturing process.

\section{PREVIOUS RESEARCH}

Franceshini and Galetto [2,6] introduced a new model to forecast the behavior of complex manufacturing plants. Their model was empirically tested in the manufacturing of automotive exhaust systems. The actual process was monitored for two months at two plants of the same company. When sufficient data was collected, the values obtained from each plant were used to predict the behavior of the other. Their study proved that the predicted and the experimental values were exhibiting similar behavior and that the learning aspects of the system could be adequately modeled. The theoretical background of their methodology is analyzed as follows.

The quality characteristics of a manufacturing process with a cumulative output of $\mathrm{q}$ units are represented over time by the cumulative number of rejected units $D(q)$. Percentage wise this property is defined by the function

$$
\mathrm{F}(\mathrm{q})=\frac{D(q)}{q}
$$

where $F(q)$ receives values between 0 and 1 . Franceshini and Galetto though advocate the use of the rate of change of $\mathrm{D}(\mathrm{q})$ for the development of their learning curve function:

$$
\mathrm{L}(\mathrm{q})=\frac{d D(q)}{d q}
$$

For a discrete manufacturing process with distinct production cycles (e.g. daily production) $\mathrm{L}(\mathrm{q})$ is computed via a finite difference equation:

$$
\mathrm{L}(\mathrm{q}) \approx \frac{\Delta D(q)}{\Delta q}
$$

(Table 1 below summarizes the procedure for the computation of the learning function for tile F in Fig. 2, a tile series which has an average shift production of approximately $930 \mathrm{~m}^{2}$ ).

Table 1 - Experimental Data of Tile F

\begin{tabular}{|c|c|c|c|c|c|c|}
\hline $\begin{array}{c}\text { Shift } \\
\text { Number } \\
\mathbf{N}\end{array}$ & $\begin{array}{c}\text { Shift } \\
\text { Production } \\
\left(\mathrm{m}^{2}\right)\end{array}$ & $\begin{array}{c}\text { Cumulative } \\
\text { Production }\left(\mathrm{m}^{2}\right) \\
\left.\mathbf{q}_{\mathbf{N}}\right)\end{array}$ & $\begin{array}{c}\text { Shift } \\
\text { Defective } \\
\text { Production }\left(\mathrm{m}^{2}\right)\end{array}$ & $\begin{array}{c}\text { Cumulative } \\
\text { Defective }\left(\mathrm{m}^{2}\right) \\
\mathbf{D}\left(\mathbf{q}_{\mathbf{N}}\right)\end{array}$ & $\begin{array}{c}\text { Cumulative } \\
\text { Defective }(\%) \\
\mathbf{F}\left(\mathbf{q}_{\mathbf{N}}\right)=\mathbf{D}\left(\mathbf{q}_{\mathbf{N}}\right) / \mathbf{q}_{\mathbf{N}}\end{array}$ & $\begin{array}{c}\text { Learning curve function } \\
\mathbf{L}\left(\mathbf{q}_{\mathbf{N}}\right)=\left[\mathbf{D}\left(\mathbf{q}_{\mathbf{N}+1}\right)-\mathbf{D}\left(\mathbf{q}_{\mathbf{N}}\right)\right] \mathbf{l q}\end{array}$ \\
\hline \hline 1 & 930 & 930 & 754 & 754 & 0,81 & 0,60 \\
\hline 2 & 930 & 1860 & 562 & 1316 & 0,71 & 0,38 \\
\hline 3 & 930 & 2790 & 358 & 1674 & 0,60 & 0,17 \\
\hline 4 & 930 & 3720 & 158 & 1832 & 0,49 & 0,13 \\
\hline 5 & 930 & 4650 & 125 & 1957 & 0,42 & 0,12 \\
\hline 6 & 930 & 5580 & 110 & 2067 & 0,37 & 0,10 \\
\hline 7 & 930 & 6510 & 93 & 2160 & 0,33 & 0,08 \\
\hline 8 & 930 & 7440 & 77 & 2237 & 0,30 & 0,07 \\
\hline 9 & 930 & 8370 & 67 & 2304 & 0,28 & 0,05 \\
\hline 10 & 930 & 9300 & 44 & 2348 & 0,25 & 0,04 \\
\hline 11 & 930 & 10230 & 40 & 2388 & 0,23 & 0,07 \\
\hline 12 & 930 & 11160 & 65 & 2453 & 0,22 & \\
\hline
\end{tabular}




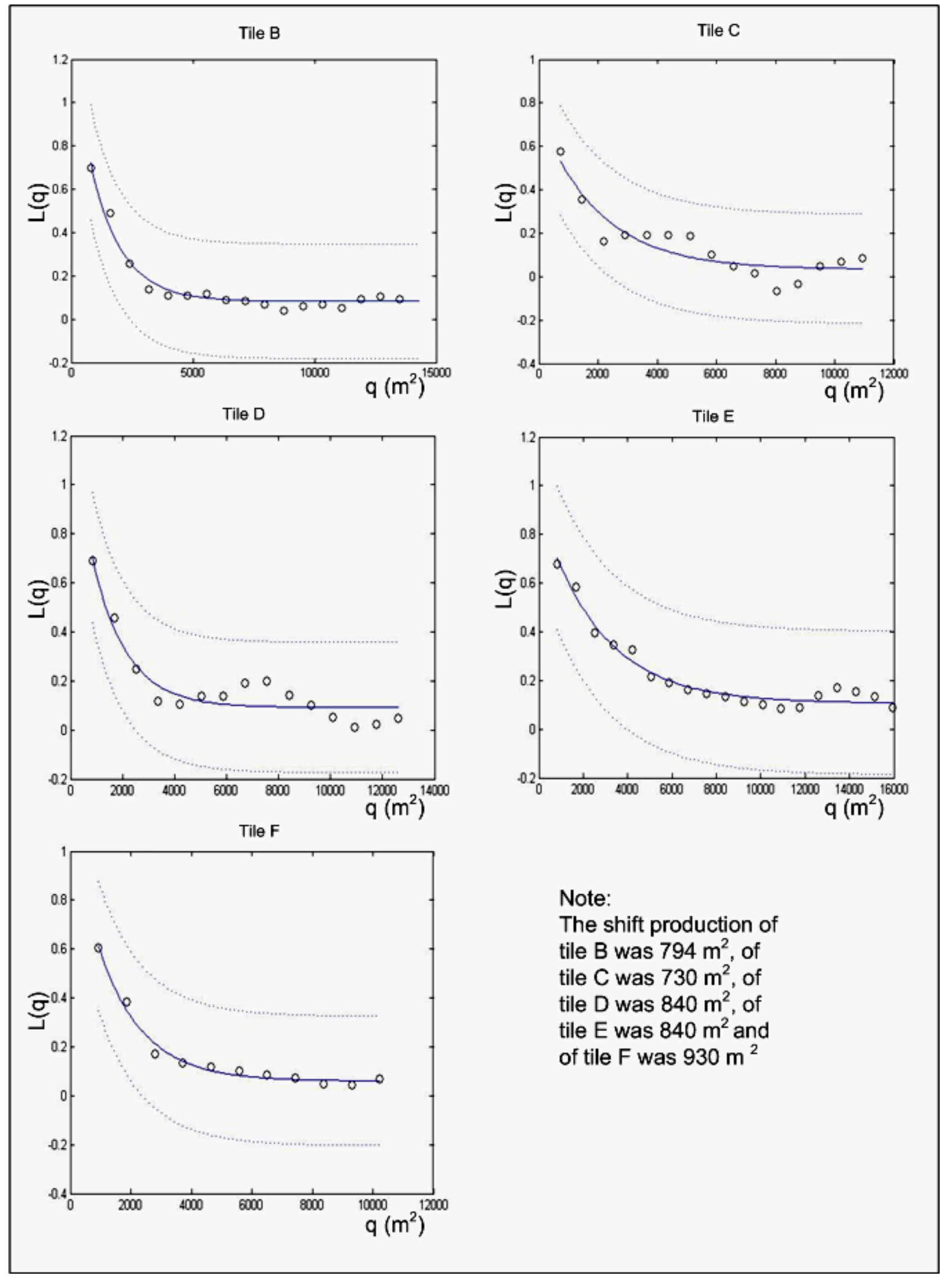

Fig. 2 Learning curves of existing products and confidence interval 
Franceshini and Galetto demonstrated through their empirical data that in the manufacturing of automotive exhaust systems the learning function exhibits a relatively smooth, exponential evolution of the form:

$$
L(q)=L_{0}+L_{1} e^{-\frac{q}{\tau}}
$$

where $\mathrm{L}_{0}, \mathrm{~L}_{1}$ and $\tau$ are constant parameters specific to the product line $[2,6]$.

The research question is of course whether their analysis is applicable to such a distinctly different manufacturing process as that of the ceramic tile industry.

\section{DATA COLLECTION AND ANALYSIS}

In the tile manufacturing industry processes unfold in a serial manner. For the purpose of this preliminary investigation, though, the entire setup is modeled via a single-input, single-output block. The corresponding learning curve refers to the production flow emerging from the quality sorting and just before palletizing.

Data was collected from the factory floor for five different tiles and for relatively small batches. The tile types were codified as B, C, D, E and F with production shift capacities of $794 \mathrm{~m}^{2}, 730 \mathrm{~m}^{2}, 840 \mathrm{~m}^{2}, 840 \mathrm{~m}^{2}$ and $930 \mathrm{~m}^{2}$ respectively. In each case, the cumulative number of rejected units $\mathrm{D}(\mathrm{q})$ was recorded as a function of the cumulative output $\mathrm{q}$ (in $\mathrm{m}^{2}$ ) and provided the basis for the computation of the learning function $\mathrm{L}(\mathrm{q})$. The processing of the data evolved along three distinct steps.

Step 1: The discrete tile data was low-pass filtered via a digital 4-point moving average filter in order to reduce noise [dot point curves in Fig. 2 and 3].

Step 2: The model used by Franceshini and Galetto in (4) was then fitted to the discrete data via non-linear regression (unweighted fit) to create the continuous $\mathrm{L}(\mathrm{q})$ learning curves for each tile run [solid line curves in Fig. 2 and 3].

Step 3: Finally, the corresponding 95\% confidence interval bands $( \pm \sigma)$ were estimated statistically using the standard deviation of the learning curve [dash line curves in Fig. 2 and 3].

A careful inspection of the summary data in Fig. 2 reveals that, as a general trend, the curve observed by Franceshini and Galetto is more or less evident for all tile runs. Admittedly, the fit is not equally good in all cases, an observation that raises the suspicion that the learning function in (4) may be too simplistic and lacking in higher-order dynamics to represent adequately a manufacturing process that is not as rigorous as that of the assembly line in an automobile plant.
The second conjecture tested in this work is whether the learning curve approach can lead to a useful forecast tool. In manufacturing terms, the question is whether historical data from past production ramp-ups can lead to meaningful assessment of new product ramp-ups in the same facility.

To address this point, the predictive capability of the tile manufacturing data at hand was tested for the ramp-up of a new tile (tile A) that has not been produced before in this facility, and thus its manufacturing process had not been completely mastered. Fig. 3 depicts the filtered data of the first run of tile A with a production shift capacity of $825 \mathrm{~m}^{2}$ as well as the bundle of confidence intervals obtained from Fig. 2 (tiles B, C, D, E, and F).

Clearly, the learning function $\mathrm{L}(\mathrm{q})$ of tile $\mathrm{A}$ is within the predicted intervals yet the bounds prescribed define a rather wide band of variation to be of practical use in the tile industry. The experiment demonstrates that the learning function can indeed describe broad systemic characteristics of a facility but further research needs to be done to identify manufacturing line and product type idiosyncrasies.

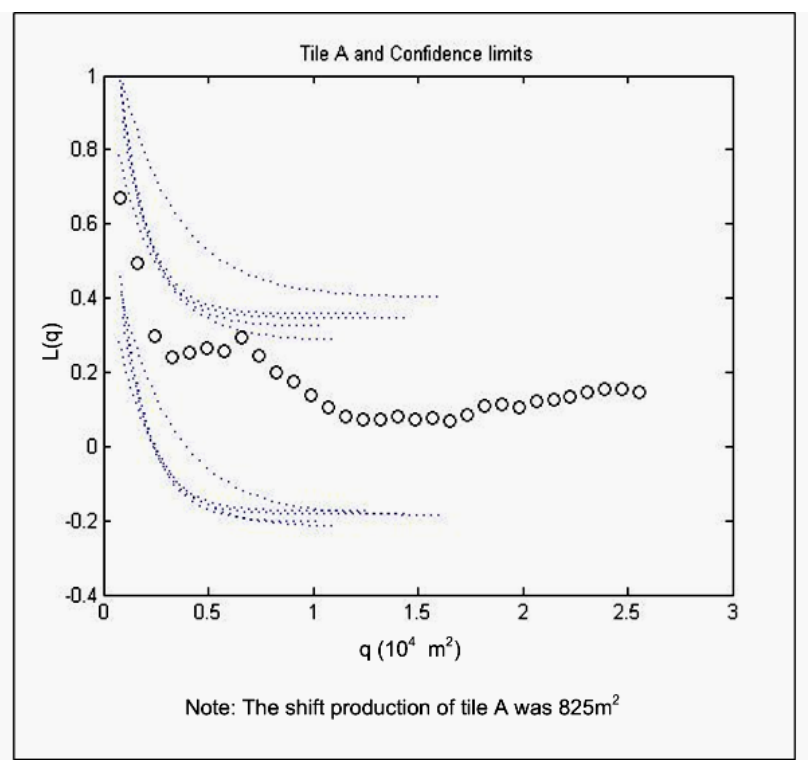

Fig. 3 Experimental behavior of a new tile

Future work should focus upon two main themes:

- Development of a learning curve function that will exhibit second- and possibly third-order dynamics to allow for more sophisticated modeling of complex manufacturing processes.

- Classification of basic and composite manufacturing processes into groups that exhibit similar learning characteristics. 


\section{CONCLUSIONS}

Thought learning curves have been used and investigated for almost seven decades now, some researchers believe that they may be used more widely in the future due to the demand for sophisticated hightechnology systems [13] and because the increased rate of product and process change means that many products are produced in the early stages of learning [14].

The ceramic tile manufacturing industry is becoming less of a mass manufacturer and more of a batch producer and is faced with a set of challenges that are novel for high-volume industries. The corresponding frequent setup and re-tooling of the line strains production and leads to considerable waste. This paper examines the premise that the learning curve phenomenon is present, in a more complex sense, when tile patterns are produced in relatively small batches and the resulting ramp-up is reminiscent of new product setup.

This basic premise is initially vetted through the Franceshini-Galetto model and the actual industrial data presented in this paper support the development of a model of line behavior that can lead to an optimum batch production level for each tile pattern.

\section{REFERENCES}

[1] K. M. Kapp, "Transforming your manufacturing organization into a learning organization," Hospital Material Management Quarterly, vol. 20, pp. 46-54, 1999.

[2] F. Fransceschini, M.Galetto, "Composition laws for learning curves of industrial manufacturing processes," Indernational Journal of Production Research, vol. 41 No 7, pp. 1431-1447, 2003.

[3] G. Fioretti, "The organizational learning curve," European Journal of Operational Research 177, 2007, pp.1375-1384.

[4] Louis E. Yelle, "The learning curve: Historical review and comprehensive surveying," Decision Sciences 10, pp. 302329, 1979.

[5] C. Terwiesch, Y. Xu, "The copy-exactly ramp-up strategy: Trading-off learning with process change," IEEE Transactions on Engineering Management, vol. 51, No.1 Feb. 2004.

[6] F. Fransceschini, M. Galetto, "An empirical investigation of learning curve composition laws for quality improvement in complex manufacturing plants," Journal of Manufacturing Technology Management, vol.15, no. 7, pp. 687-699, 2004.

[7] G. A. F. Seber, C. J. Wild, 1989, Nonlinear Regression, Wiley Series in Probability and Mathematical Statistics, Wiley, New York, NY.

[8] J. Vits, L. Gelders, L. Pintelon, "Production process changes: A dynamic programming approach to manage effective capacity and experience," International Journal of Production Economics 104, pp. 473-481, 2006

[9] H. Almgren, "Pilot production and manufacturing start-up: the case of Volvo S80," Indernational Journal of Production Research, vol 38, pp.4577-4588, 2000.

[10] C. Terwiesch, R. Bohn, "Learning and process improvement during production ramp-up," International Journal of Production Economics 70, pp. 1-19, 2001.
[11] C. Terwiesch, R. Bohn, K. S. Chea, "International product transfer and production ramp-up: a case study from the data storage industry," R\&D Management 31, 4, pp. 435-451, 2001.

[12] V. Krishnan, K. T. Ulrich, "Production development decisions: A review of the literature," Management Science, vol47, no. 1, pp. 1-21, 2001.

[13] G. J. Steven, "The learning curve: from aircraft to spacecraft?," Management Accounting, 77 (5), pp. 64-65, 1999.

[14] M. Y. Jader, M. Bonney, "Economic manufacturing quantity (EMQ) model with lot-size dependent learning and forgetting rates," International Journal of Production Economics, vol. 108, pp. 359-367, 2007. 